Discrete Comput Geom 32:601-621 (2004)

DOI: $10.1007 / \mathrm{s} 00454-004-1140-4$

Geometry

\title{
The $E_{t}$-Construction for Lattices, Spheres and Polytopes*
}

\author{
Andreas Paffenholz and Günter M. Ziegler \\ Institut für Mathematik, MA 6-2, Technische Universität Berlin, \\ D-10623 Berlin, Germany \\ \{paffenholz,ziegler\}@math.tu-berlin.de
}

\begin{abstract}
We describe and analyze a new construction that produces new Eulerian lattices from old ones. It specializes to a construction that produces new strongly regular cellular spheres (whose face lattices are Eulerian). The construction does not always specialize to convex polytopes; however, in a number of cases where we can realize it, it produces interesting classes of polytopes. Thus we produce an infinite family of rational 2-simplicial 2-simple 4-polytopes, as requested by Eppstein et al. [6]. We also construct for each $d \geq 3$ an infinite family of $(d-2)$-simplicial 2-simple $d$-polytopes, thus solving a problem of Grünbaum [9].
\end{abstract}

\section{Introduction}

Eulerian lattices provide an interesting, entirely combinatorial model for the combinatorics (face lattices) of convex polytopes. Although the concepts have been around for a long time (Eulerian posets were formalized by Stanley [17] in 1982, but the ideas may be traced back at least to Klee's paper [13] from 1964), there is surprisingly little systematic knowledge and development of Eulerian lattices, despite a number of extensive recent studies of (the flag vectors of) Eulerian posets; see [20]. A complete list of linear relations for the flag vectors of polytopes, spheres and lattices was given by Bayer and Billera in [1], where they proved their generalized Dehn-Sommerville relations.

In this paper we present a combinatorial construction $E_{t}$ for Eulerian lattices which specializes to the setting of "strongly regular spheres." Here the parameter $t$ is an integer

* The research by the first author was supported by the Deutsche Forschungsgemeinschaft within the European graduate program "Combinatorics, Geometry, and Computation" (No. GRK 588/2). The second author was partially supported by Deutsche Forschungsgemeinschaft, via the DFG Research Center "Mathematics in the Key Technologies" (FZT86), the Research Group "Algorithms, Structure, Randomness" (Project ZI 475/3), a Leibniz grant (ZI 475/4) and by the German Israeli Foundation (G.I.F.). 
between 0 and $\ell-2$, where $\ell$ is the length of the lattice, and denotes the dimension (rank plus one) of the elements that will correspond to coatoms of the new lattice. The $E_{t}$ construction may be more intuitive when applied to cellular spheres, but Eulerian lattices provide a simple axiomatic setting in which we can most easily analyze the construction and derive its properties. Thus we start with lattices (Section 1), and in a second step transfer the results to spheres and give a geometric interpretation of the construction (Section 2). In some cases this construction can be performed in the setting of convex polytopes, and can then be used to construct interesting new classes of such (Section 3):

1. We provide the first infinite family of rational 2-simplicial 2-simple 4-polytopes.

2. We present for each $d \geq 3$ an infinite family of $(d-2)$-simplicial 2-simple $d$-polytopes.

The first class solves a problem posed by Eppstein et al. ([6], compare also [23]). They provided an infinite family of 2-simplicial 2-simple 4-polytopes, but their construction is quite rigid and produces nonrational coordinates. In contrast, our construction will allow great flexibility for the actual choice of coordinates for a geometric realization of the polytopes in this family. Thus we can also derive a number of consequences for the $f$-vector classification problem for four-dimensional polytopes (Section 4). The second class of polytopes solves a problem of Grünbaum [9, Problem 9.7.7(iii)] and thus substantiates claims by Perles and Shephard [9, p. 170] and by Kalai [12, Problem 20.5.19].

Our construction contains the method of Eppstein et al. as a special case: if we choose $t=1$ and apply $E_{1}$ to the face lattice of a simplicial 4-polytope $P$ whose edges are tangent to the unit sphere, then we obtain the face lattice of a 2-simplicial 2-simple polytope which they call $E(P)$. Thus the second class of examples displayed above is obtained by a generalization of the approach from [6] to higher dimensions.

In contrast, the construction of the first class relies on the analysis of the dual construction to $E_{1}$, which we describe as "vertex truncation" in Section 3.1. The special case where $P$ is a regular polytope appears in the literature in the context of the construction of regular and "uniform" polytopes: here our vertex truncation can be seen as an instance of "Wythoff's construction" as described by Coxeter [4, p. 147], [5], while a special case of the dual $E_{t}$-construction appears in Gévay's work [7], [8].

\section{Eulerian Lattices}

We refer to [18] for general terminology and background about posets, lattices and their enumerative combinatorics. All the posets we consider are finite. A bounded poset is required to have a unique maximal element $\hat{1}$ and a unique minimal element $\hat{0}$. A poset $L$ is a lattice if any two elements $y, y^{\prime} \in L$ have a unique minimal upper bound $y \vee y^{\prime}$ and a unique maximal lower bound $y \wedge y^{\prime}$, known as the join and the meet of $y$ and $y^{\prime}$, respectively. By $\bigvee A$ and $\bigwedge A$ we denote the join, resp. the meet, of a subset $A \subseteq L$. A lattice is complemented if for every element $x$ there is an element $x^{\prime}$ with $x \wedge x^{\prime}=\hat{0}$ and $x \vee x^{\prime}=\hat{1}$.

A bounded poset is graded if all its maximal chains have the same length. Every graded poset comes with a rank function $r: L \rightarrow \mathbb{Z}$, normalized such that $r(\hat{0})=0$. The length of a graded poset is given by $r(\hat{1})$. By $L_{i}$ we denote the set of all elements of rank $i+1$ 
in $L$, for $-1 \leq i \leq r(\hat{1})-1$. We also talk about the dimension $\operatorname{dim}(x):=r(x)-1$ of an element $x \in L$ : This is motivated by the important situation when $L$ is the face lattice of a polytope, and $x \in L_{i}$ corresponds to a face of dimension $i$. In this case we write $d:=r(\hat{1})-1$ for the dimension of the polytope.

By $f_{i}(L)=\left|L_{i}\right|$ we denote the number of elements of rank $i+1$ (for $-1 \leq i \leq d$ ), while $f_{i j}(L)$, for $-1 \leq i \leq j \leq d$, denotes the number of pairs of elements $x \in L_{i}$ and $y \in L_{j}$ with $x \leq y$. If $L$ is a bounded poset, we denote by $\bar{L}:=L \backslash\{\hat{0}, \hat{1}\}$ its proper part. The minimal elements of $\bar{L}$, i.e. the elements of $L$ of $\operatorname{rank} 1$, are the atoms of $L$; similarly the maximal elements of $\bar{L}$, i.e. the elements of $L$ of rank $d$, are the coatoms of $L$. By $L^{\mathrm{op}}$ we denote the opposite of a poset $L$, that is, the same set, but with reversed order relation.

Definition 1.1. A graded poset $L$ of length $d+1$ is Eulerian if every interval $[x, y]$ with $x<y$ has the same number of elements of odd and even rank.

This definition is equivalent to the "usual" definition [18, p. 122], which requires that $\mu(x, y)=(-1)^{r(y)-r(x)}$ for all $x \leq y$, where $\mu$ is the Möbius function [18, Exercise 3.69(a)].

Definition 1.2 (The $E_{t}$-Construction for Graded Posets). Let $L$ be a graded poset of length $d+1$, and let $t$ be any integer in the range $0 \leq t \leq d-1$.

The $E_{t}$-construction applied to $L$ yields the poset $E_{t}(L)$ that consists of the following subsets of $L$, ordered by reversed inclusion:

- the empty set,

- the one element sets $\{y\}$ for $y \in L_{t}$ and

- the intervals $[x, z] \subseteq L$ such that some $y \in L_{t}$ satisfies $x<y<z$.

The rank function on $E_{t}(L)$ is given by

$$
\rho(b)= \begin{cases}r(x)+d+1-r(z) & \text { for } b=[x, z], \quad r(x)<t+1<r(z), \\ d & \text { for } b=\{y\}, \quad y \in L_{t}, \\ d+1 & \text { for } b=\emptyset .\end{cases}
$$

Thus $E_{t}(L)$ is again a graded poset of length $d+1$. (Compare Fig. 1.) Its coatoms are the one-element sets $\{y\}, y \in L_{t}$, its atoms are the intervals $[x, \hat{1}]$ (for $1 \leq t \leq d-1$ )
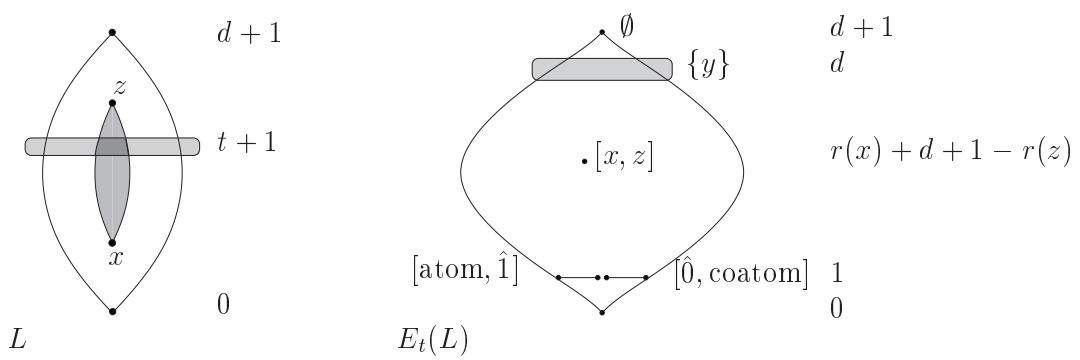

Fig. 1. Combinatorial construction of $E_{t}(L)$. 
and $[\hat{0}, z]$ (for $0 \leq t \leq d-2$ ), where the $x$ and $z$ are the atoms, resp. coatoms, of $L$. The $f$-vector is

$$
f_{k}\left(E_{t}(L)\right)= \begin{cases}\sum_{i, j} f_{i j}(L) & \text { for } \quad-1 \leq k<d-1, \\ f_{t}(L) & \text { for } k=d-1, \\ 1 & \text { for } k=d,\end{cases}
$$

where the sum in the above formula is over all pairs $i, j$ in the range $-1 \leq i<t<j \leq d$ with $j-i=d-k$.

In the following we are mostly interested in Eulerian lattices, that is, in Eulerian posets which at the same time satisfy the lattice property. If $L$ is a lattice, then the set $E_{t}(L)$ can more compactly be described as

$$
E_{t}(L):=\left\{[\bigwedge A, \bigvee A]: A \subseteq L_{t}\right\}
$$

again ordered by reversed inclusion. In this case $E_{t}(L)$ is again a lattice: the join and meet operations in $E_{t}(L)$ are given by

$$
[x, z] \wedge\left[x^{\prime}, z^{\prime}\right]=\left[x \wedge x^{\prime}, z \vee z^{\prime}\right], \quad[x, z] \vee\left[x^{\prime}, z^{\prime}\right]=\left[x \vee x^{\prime}, z \wedge z^{\prime}\right] .
$$

This follows from the fact that every interval $[x, z]$ is itself an Eulerian lattice, hence it has a nonzero Möbius number, hence it is complemented, and the join of its atoms is the maximal element and the meet of the coatoms is the minimal element (see Corollary 3.9.5 of [18]).

Remark. Even if $L$ is an Eulerian poset that is not a lattice, $E_{t}(L)$ may still be a lattice.

Example 1.3. Let $L$ be a graded poset of length $d+1$. For $t=0$ we obtain $E_{0}(L) \cong$ $L^{\mathrm{op}}$. For $t=d-1$ we get $E_{d-1}(L) \cong L$. Thus the "boundary cases" of $t=0$ and $t=d-1$ are not interesting, and hence they are excluded from some of the following discussions without loss of interesting effects. We also note that

$$
E_{t}(L)=E_{d-1-t}\left(L^{\mathrm{op}}\right),
$$

so we can derive the same posets/lattices from $L$ and from $L^{\text {op }}$.

Theorem 1.4. Let $L$ be an Eulerian poset of length $d+1$ and let $0 \leq t \leq d-1$. Then $E_{t}(L)$ is an Eulerian poset of the same length $d+1$.

Proof. This is true for $t=0$ and for $t=d-1$, which includes all possible $t$-values for $d \leq 2$. Thus we may use induction on the length of $L$.

First we show that all proper intervals in $E_{t}(L)$ are Eulerian. Indeed, for any element $[x, z] \in E_{t}(L),(x, z) \neq(\hat{0}, \hat{1})$, the upper interval $[[x, z], \hat{1}]$ of $E_{t}(L)$ is isomorphic to $E_{t^{\prime}}([x, z])$ for $t^{\prime}=t-r(x)$; hence all proper upper intervals in $E_{t}(L)$ are produced by the $E_{t}$-construction from Eulerian posets of smaller length, so they are Eulerian by induction. 
Similarly, if $[x, z]$ is an element of rank at most $d-1$ in $E_{t}(L)$, that is, an interval of $L$ with $x<y<z$ for some $y \in L_{t}$, then the lower interval $[0,[x, z]]$ of $E_{t}(L)$ is isomorphic to $[\hat{0}, x] \times[z, \hat{1}]^{\mathrm{op}}$, which is Eulerian since $L$ is Eulerian. If $\{y\}$ is a coatom of $E_{t}(L)$, for $y \in L_{t}$, then the lower interval $[\hat{0},\{y\}]$ is isomorphic to $\left(L_{<y}\right) \times\left(L_{>y}\right)^{\mathrm{op}} \uplus \hat{1}$. Thus it is a "reduced product" and this operation is known [18, Exercise 3.69(d)] [19, p. 485] and easily checked to preserve the Eulerian property; compare Section 6 of [21].

Finally, we have to see that $E_{t}(L)$ itself has the same number of odd and even rank elements. For this we may use the $f$-vector of $E_{t}(L)$, as computed above. Every interval $[\hat{0}, z]$ is Eulerian. Thus for $0 \leq j \leq d-1$ and all $z \in L_{j}$ we have $\sum_{i=-1}^{j}(-1)^{i} f_{i}([\hat{0}, z])=$ 0 , which by summing over all $z \in L_{j}$ yields $\sum_{i=-1}^{j}(-1)^{i} f_{i j}=0$, that is,

$$
\sum_{i=-1}^{t-1}(-1)^{i} f_{i j}=-\sum_{i=t}^{j}(-1)^{i} f_{i j}
$$

for $j \geq t \geq 0$. This is one of the generalized Dehn-Sommerville equations [1]. A similar argument for upper intervals shows that

$$
\sum_{j=i}^{d}(-1)^{j} f_{i j}=(-1)^{d} \delta_{i d}
$$

for $i \leq d$. With these two equations, we can compute

$$
\begin{aligned}
& \sum_{k=-1}^{d}(-1)^{d-k} f_{k}\left(E_{t}(L)\right) \\
& \quad=1-f_{t}+\sum_{i=-1}^{t-1} \sum_{j=t+1}^{d}(-1)^{j-i} f_{i j} \quad=1-f_{t}+\sum_{j=t+1}^{d}(-1)^{j} \sum_{i=-1}^{t-1}(-1)^{i} f_{i j} \\
& \quad \stackrel{(2)}{=} 1-f_{t}-\sum_{j=t+1}^{d}(-1)^{j} \sum_{i=t}^{j}(-1)^{i} f_{i j}=1-\sum_{j=t}^{d}(-1)^{j} \sum_{i=t}^{j}(-1)^{i} f_{i j} \\
& =1-\sum_{i=t}^{d}(-1)^{i} \sum_{j=i}^{d}(-1)^{j} f_{i j} \quad \stackrel{(3)}{=} 1-\sum_{i=t}^{d}(-1)^{i}(-1)^{d} \delta_{i d}=0 .
\end{aligned}
$$

Alternatively, one may argue from Theorem 2.1 in the next section: since the order complexes of $L$ and of $E_{t}(L)$ are homeomorphic, they must have the same Euler characteristic, which is the Möbius function of $L$, resp. $E_{t}(L)$, which is what we need for $(x, z)=(\hat{0}, \hat{1})$.

Definition 1.5. Let $L$ be an Eulerian lattice of length $d+1$, and let $0 \leq k, h \leq d-1$.

- $L$ is boolean if it is isomorphic to the face lattice of a $d$-simplex.

- $L$ is $k$-simplicial if all intervals $[\hat{0}, z]$ with $r(z)=k+1$ are boolean.

- It is $h$-simple if all intervals $[x, \hat{1}]$ with $r(x)=d-h$ are boolean.

- $L$ is simple, resp. simplicial, if it is $(d-1)$-simple, resp. $(d-1)$-simplicial.

A $(k, h)$-lattice $L$ is a $k$-simplicial and $h$-simple Eulerian lattice. 
Every $k$-simplicial Eulerian lattice is also $(k-1)$-simplicial for $k>0$. All Eulerian lattices are 1 -simplicial. The property " $k$-simplicial" is dual to " $k$-simple"; thus every Eulerian lattice is also 1-simple. $L$ is a $(k, h)$-lattice if and only if $L^{\mathrm{op}}$ is an $(h, k)$-lattice.

Remark. Every $(k, h)$-lattice of length $d+1$ with $k+h>d$ is boolean. (In particular, any $k$-simplicial $h$-simple $d$-polytope with $k+h>d$ is a simplex.) The proof, also in the generality of Eulerian lattices, is a straightforward extension of the argument for simple and simplicial polytopes (see p. 65 of [9] and p. 23 of [22]).

Any Eulerian lattice is graded and complemented, so by induction on the length it follows that every Eulerian lattice $L$ of length $\ell$ has at least $\left(\begin{array}{l}\ell \\ i\end{array}\right)$ elements of rank $i$. Furthermore, if equality holds for some $i, 0<i<\ell$, then $L$ is boolean. In particular, any Eulerian lattice of length $\ell$ has at least $\ell$ atoms, with equality only if $L$ is boolean, and similarly for coatoms. We rely on this criterion in the proof of the following characterization of Eulerian lattices $L$ for which $E_{t}(L)$ is $k$-simplicial and $h$-simple.

Theorem 1.6. Let $L$ be an Eulerian lattice of length $d+1$ and let $1 \leq t \leq d-2$.

(1) For $0 \leq k \leq d-2$, the lattice $E_{t}(L)$ is $k$-simplicial if and only if $L$ is $s$-simplicial and $r$-simple for $s=\min \{k, t-1\}$ and $r=\min \{k, d-t-2\}$.

$\left(1^{\prime}\right)$ The lattice $E_{t}(L)$ is never $(d-1)$-simplicial.

(2) The lattice $E_{t}(L)$ is 2-simple if and only if every interval $[\bar{x}, \bar{z}] \subseteq L$ with $r(\bar{x})=$ $t-1$ and $r(\bar{z})=t+3$ is boolean.

In particular, this is the case if $L$ is $(t+2)$-simplicial or $(d-t+1)$-simple.

$\left(2^{\prime}\right)$ The lattice $E_{t}(L)$ is never 3-simple.

Proof. (1) The elements of rank at most $k+1 \leq d-1$ in $E_{t}(L)$ are the intervals of the form $a=[x, z] \subseteq L$ with $r(x) \leq t, r(z) \geq t+2$ satisfying $r(x)+(d+1)-r(z)=$ $\rho([x, z]) \leq k+1$. An element $x \in L$ appears as the lower end of such an interval $[x, z]$ if and only if $0 \leq r(x) \leq \min \{k+1, t\}$; similarly, this concerns all elements $z \in L$ of corank $0 \leq d+1-r(z) \leq \min \{k+1, d-t-1\}$.

The atoms of $E_{t}(L)$ below $a=[x, z]$ are given by both the atoms of $L$ below $x$, whose number is at least $r(x)$, and the coatoms of $L$ above $z$, whose number is at least $d+1-r(z)$. So the interval has at least $r(x)+d+1-r(z)=\rho([x, z])$ atoms, with equality if and only if the intervals $[\hat{0}, x]$ and $[z, \hat{1}]$ of $L$ are both boolean.

Thus all lower intervals $[\hat{0}, a]$ of rank $\leq k+1$ in $E_{t}(L)$ are boolean if and only if all intervals $[\hat{0}, x]$ and $[z, \hat{1}]$ are boolean for $r(x) \leq \min \{k+1, t\}$, resp. $d+1-r(z) \leq$ $\min \{k+1, d-t-1\}$.

$\left(1^{\prime}\right)$ An analysis as for (1) shows that for any element $\{y\}$ of rank $d$ in $E_{t}(L)$, that is, for $y \in L_{t}$, there are at least $t+1$ atoms in $L$ below $y$ and at least $d-t$ coatoms in $L$ above $y$; thus there are at least $(t+1)+(d-t)=d+1$ atoms below $\{y\}$ in $E_{t}(L)$ : too many.

(2) $E_{t}(L)$ is 2-simple if all intervals $[b, \hat{1}] \subset E_{t}(L)$ with $b=[x, z] \subset L, \rho(b)=$ $d-2$, are boolean, that is, they have three atoms or coatoms. This is the case if and only if every interval $b=[x, z] \subset L, r(x)<t+1<r(z)$, of length 3 contains precisely three 
elements of rank $t+1$. This is equivalent to the condition that every length 4 interval $[\bar{x}, \bar{z}]$ with $r(\bar{x})=t-1$ and $r(\bar{z})=t+3$ is boolean. In terms of the usual flag vector notation, this can be numerically expressed as $f_{t-2, t, t+2}(L)=6 f_{t-2, t+2}(L)$.

$\left(2^{\prime}\right)$ Similarly, for $E_{t}(L)$ to be 3 -simple we would need that every interval $[x, z]$ in $L$ of length 4 with $r(x)<t+1<r(z)$ and $r(z)=r(x)+4$ contain exactly four elements of rank $t+1$. This is impossible for the case where $r(x)=t-1$ and $r(z)=t+3$, where the interval $[x, z]$ has at least six elements in its "middle level" (that is, of rank $t+1$ ) by the Eulerian condition.

Corollary 1.7. For $d \geq 3$ and any simplicial Eulerian lattice L of length $d+1$ the lattice $E_{d-3}(L)$ is $(d-2)$-simplicial and 2-simple.

\section{CW Spheres}

The order complex of a bounded poset $L$ is the abstract simplicial complex of chains in its proper part $\bar{L}$, denoted by $\Delta(\bar{L})$. By $\|\bar{L}\|$ we denote the geometric realization of the order complex of the poset $\bar{L}$.

A CW complex is regular if all its closed cells are embedded, that is, if the attaching maps of the individual cells make no identifications on the boundary. In the following, all cell complexes are finite regular $\mathrm{CW}$ complexes. A cell complex is strongly regular if the following "intersection property" [2, Problem 4.47, p. 223] holds: The intersection of any two cells is a single closed cell (which may be empty). This holds if and only if not only the cells but also all the stars of vertices are embedded. For example, the boundary complex of any convex $d$-polytope is a strongly regular $\mathrm{CW}(d-1)$-sphere.

The main property of PL spheres and manifolds (see [10] for the definitions) we need is the following: They come with regular cell decompositions such that not only the boundary of each cell is a sphere (of the appropriate dimension), but also the links of all faces are genuine spheres (not only homology spheres, as in general triangulated or cellular manifolds [2, Proposition 4.7.21]). Equivalently, in the face poset of the cell decomposition, augmented by a maximal element $\hat{1}$, not only the order complexes of the lower intervals $[\hat{0}, x]$ with $x<\hat{1}$ are spheres, but the same is true for all intervals $[x, y]$, with the only possible exception of $[\hat{0}, \hat{1}]$, whose order complex is homeomorphic to the manifold in question.

Finally, a bounded, graded poset $P$ is the face poset of a regular $\mathrm{CW}$ sphere if and only if the order complex of every interval $[\hat{0}, x], x \in P$, is homeomorphic to a sphere of dimension $r(x)-2$; see Proposition 4.7.23 of [2].

The following is similar to (simpler) results and proofs for interval posets in [21].

Theorem 2.1. Let $L$ be a graded poset of length $d+1$. Then $L$ and $E_{t}(L)$ are $P L$ homeomorphic:

$$
\|\bar{L}\| \cong\left\|\overline{E_{t}(L)}\right\| .
$$

Proof. We verify that $\Delta\left(\overline{E_{t}(L)}\right)$ is a subdivision of $\Delta(\bar{L})$, and give explicit formulas for the subdivision map and its inverse. (Compare this with Sections 4 and 5 of [21].) 
A canonical map $\pi:\left\|\Delta\left(\overline{E_{t}(L)}\right)\right\| \longrightarrow\|\Delta(\bar{L})\|$, linear on the simplices, is given by its values on the vertices:

$$
\begin{array}{rlll}
\{y\} & \longmapsto y & & \text { for } y \in L_{t}, \\
{[x, z]} & \longmapsto \frac{1}{2} x+\frac{1}{2} z & \text { for } \hat{0}<x<y<z<\hat{1}, \quad y \in L_{t}, \\
{[x, \hat{1}]} & \longmapsto x & \text { for } \hat{0}<x<y<\hat{1}, \quad y \in L_{t}, \\
{[\hat{0}, z]} & \longmapsto z & \text { for } \hat{0}<y<z<\hat{1}, \quad y \in L_{t} .
\end{array}
$$

This map is well-defined and continuous. Its inverse, a subdivision map, may be described as follows: Any point of $\Delta(\bar{L})$ is an affine combination of elements on a chain in $\bar{L}$, so it may be written as

$$
\mathbf{x}: \lambda_{1} x_{1}<\cdots<\lambda_{t} x_{t}<\lambda_{t+1} y_{t+1}<\lambda_{t+2} z_{t+2}<\cdots<\lambda_{d} z_{d}
$$

with $\lambda_{i} \geq 0$ and $\sum_{i} \lambda_{i}=1$. We set $x_{0}:=\hat{0}$ and $z_{d+1}:=\hat{1}$, with $\lambda_{0}:=1$ and $\lambda_{d+1}:=1$. Now the above point is mapped by $\pi^{-1}$ to

$$
\pi^{-1}(\mathbf{x})=\lambda_{t+1}\left\{y_{t+1}\right\}+\sum_{1 \leq i<t, t+1<j \leq d} 2 \alpha_{i, j}\left[x_{i}, z_{j}\right]+\sum_{\substack{i=0, t+1<j \leq d \text { or } \\ 1 \leq i \leq t, j=d+1}} \alpha_{i, j}\left[x_{i}, z_{j}\right],
$$

where the coefficients $\alpha_{i, j}$ are given by

$$
\alpha_{i, j}= \begin{cases}\min \{f(i), g(j)\}-\max \{f(i+1), g(j-1)\} & \text { if this is } \geq 0 \\ 0 & \text { otherwise }\end{cases}
$$

with

$$
\begin{aligned}
& f(i):=\lambda_{i}+\lambda_{i+1}+\cdots+\lambda_{t} \quad \text { for } \quad 0 \leq i \leq t+1, \quad \text { and } \\
& g(j):=\lambda_{t+2}+\cdots+\lambda_{j-1}+\lambda_{j} \quad \text { for } \quad t+1 \leq j \leq d+1
\end{aligned}
$$

(with $f(t+1)=g(t+1)=0)$. Refer to Fig. 2 for illustration.

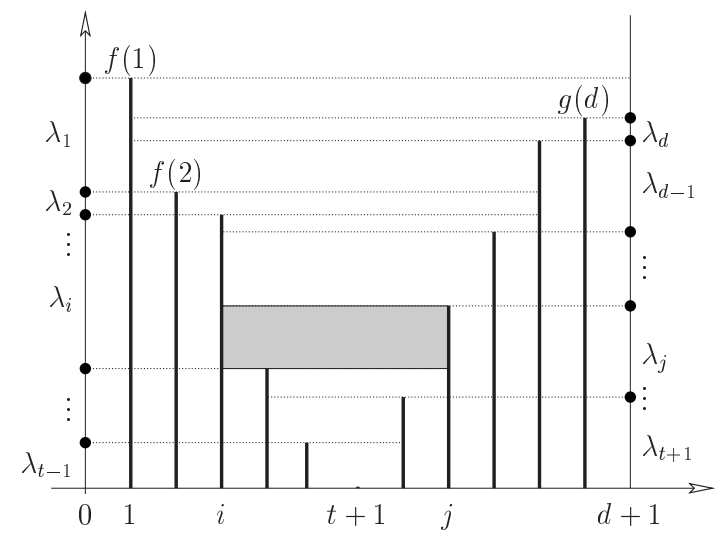

Fig. 2. Sketch for the proof of Theorem 2.1. The height of the shaded rectangle indicates the size of the coefficient $\alpha_{i, j}$. 
Theorem 2.2. If $L$ is the face poset of a regular $C W P L$ sphere or PL manifold, then so is $E_{t}(L)$.

Proof. By Theorem 1.4 and its proof, using the PL property, we get the cell complex. By Theorem 2.1, this cell complex is homeomorphic to $\|\bar{L}\|$.

The following is an easy consequence of Theorem 2.2 and Corollary 1.7.

Corollary 2.3. For $d \geq 3$ and for any strongly regular simplicial PL-sphere $S$ of dimension $d-1$, the PL-sphere $E_{d-3}(S)$ is $(d-2)$-simplicial and 2-simple.

\section{Polytopes}

We refer to [9] and [22] for background on polytopes. The boundary of any polytope $P$ naturally carries the structure of a strongly regular CW PL-sphere. Thus we can apply the $E_{t}$-construction to $\partial P$ and get a new PL-sphere $E_{t}(P)$. As mentioned earlier, it is not at all clear that $E_{t}(P)$ can be realized as a polytope, for any given convex polytope $P$ and for given $t$. However, in the main part of this section we present and analyze two techniques that do yield infinite families of interesting $E_{t}$-polytopes:

- The first construction is surprisingly simple; it produces the first infinite families of rational 2-simplicial 2-simple 4-polytopes.

- The second construction is an extension of the $E$-construction of Eppstein et al. [6] to higher dimensions; it produces for each $d \geq 3$ infinitely many $(d-2)$-simplicial 2 -simple $d$-polytopes; as far as we can see this is new for $d>4$, and confirms assertions of Grünbaum [9, pp. 169-170] and Kalai [12, Problem 20.5.19].

Furthermore, we then survey the previously known examples of $(d-2)$-simplicial 2simple polytopes, and demonstrate that virtually all of them can be realized as instances of the $E_{t}$-construction or of its dual.

\subsection{Realizations via Vertex Truncation}

The following simple construction-if it can be realized-produces a polytope that is dual to $E_{1}(P)$. A very special case of it turns up already in Gosset's 1897 construction of the 24-cell. A slightly more general version appears in Coxeter's book in the construction of the three special four-dimensional regular polytopes [4, pp. 145-164]: He considers "truncation" of polytopes by hyperplanes defined by all vertex vectors of a centered regular $d$-polytope and thinks of this as a continuous process having $d-1$ interesting stages in which the cutting hyperplanes intersect in the centroids of the $k$-faces, for $1 \leq k \leq d-1$.

Definition 3.1 (Vertex Truncation). Let $P$ be any $d$-polytope, $d \geq 3$. A vertex $v$ of $P$ is truncated if we intersect $P$ with a closed half-space that does not contain $v$, but contains all other vertices of $P$ in its interior. 
Vertex truncation (that is, "truncating a single vertex") results in a new polytope with one additional facet, and with new vertices $u_{e}$ corresponding to the edges of $P$ that contain the vertex $v$ of $P$ that has been truncated.

Definition 3.2 (Truncatable Polytopes). $\quad \mathrm{A} d$-polytope $P$ is truncatable if all its vertices can be truncated simultaneously in such a way that one single (relative interior) point remains from each edge of $P$. The resulting polytope from such a construction is denoted $D_{1}(P)$.

Whether this construction can be performed depends on the realization of $P$, and on a careful choice of the hyperplanes/half-spaces that truncate the vertices of $P$ - see the case when $P$ is a possibly nonregular octahedron. If it can be performed, the resulting polytope is usually not uniquely determined - see the case when $P$ is a simplex. However, the following proposition establishes that if the construction can be performed, then the combinatorial type of $D_{1}(P)$ is uniquely determined, and that it is of interest for our investigations.

Proposition 3.3. Let $P$ be any $d$-polytope, $d \geq 3$. If $P$ is truncatable, then the dual $D_{1}(P)^{*}$ of the resulting polytope realizes $E_{1}(P)$.

Proof. The polytope $D_{1}(P)$ has two types of facets: First there are the facets $F^{\prime}$ that are obtained by vertex truncation from the facets $F$ of $P$. (Here we use that $d \geq 3$.) Secondly, there are the "new" facets $F_{v}$ that result from truncating the vertices of $P$. The intersection of any two new facets $F_{v}$ and $F_{w}$ is empty if $v$ and $w$ are not adjacent in $P$. Otherwise the intersection $F_{v} \cap F_{w}$ consists of one single point, the new vertex $u_{e}$ given by the edge $e=(v, w)$. The vertices of $D_{1}(P)$ are given by $u_{e}$, in bijection with the edges $e$ of $P$.

Thus to check that $D_{1}(P)$ has the right combinatorics, we only check the correct vertex-facet incidences: $u_{e}$ lies on $F^{\prime}$ if and only if $e$ is an edge of $F$, and $u_{e}$ lies on $F_{v}$ if and only if $e$ is adjacent to $v$. This amounts to the reversed atom-coatom incidences of $E_{1}(P)$.

For the goal of our considerations-producing $(d-2)$-simplicial 2-simple $d$-polytopes-this construction is only interesting in dimensions $d=3$ and $d=4$, where we can apply it to simplicial polytopes, trying to get 2-simple and (for $d=4$ ) 2-simplicial polytopes. In all other dimensions we would have to apply it to a 3-simplicial $(d-3)$ simple $d$-polytope to obtain a $(d-2)$-simplicial and 2-simple one, and for large $d$ we know only two such examples in every dimension (the simplex and the half-cube $N^{d}$; see Section 3.3). So from now on we are mainly interested in classes of three- and four-dimensional polytopes.

A first obvious class of truncatable polytopes is the following:

Proposition 3.4. If a $d$-polytope $P, d \geq 3$, is realized such that all edges are tangent to a $(d-1)$-dimensional sphere $S$, then it is truncatable. 
Proof. Consider any vertex $v$ of the polytope $P$ and consider the tangent cone $C$ to $S$ with apex $v$. Then the intersection of $C$ with $S$ is a $(d-2)$-dimensional sphere $S^{\prime}$. As all edges adjacent to $v$ are tangent to $S$, they are contained in $C$ and the point of tangency must be contained in $S^{\prime}$. Thus we can cut with the hyperplane defined by $S^{\prime}$.

By the Koebe-Andreev-Thurston circle packing theorem [22, Theorem 4.12], every 3-polytope has a realization that is edge-tangent and hence truncatable. In dimension 4, edge-tangent polytopes $P$ were constructed and $E_{1}(P)=D_{1}(P)^{*}$ obtained by Eppstein et al. [6], so our construction subsumes all their examples. However, we now show that an "edge-tangency" is not necessary for the realization of $D_{1}(P)$ or $E_{1}(P)$.

A stacked polytope [12] is obtained from a simplex by repeatedly adding a new vertex "beyond" a facet, that is, by gluing a simplex onto a facet.

By construction, any stacked $d$-polytope is simplicial and the vertex added last is adjacent to precisely $d$ edges.

Theorem 3.5. Every stacked d-polytope $(d \geq 3)$ has a geometric realization that is truncatable.

Proof. Let $P_{n}^{d}$ denote a stacked $d$-polytope $(d \geq 3)$ with $n+d+1$ vertices, i.e. a $d$-simplex that has been stacked $n$ times, $n \geq 0$. (Compare Fig. 3.) We do not care about the actual choice of facets above which we have placed a vertex, so that $P_{n}^{d}$ might denote many combinatorially distinct polytopes. Obviously $P_{0}^{d}$ is truncatable. Now let $P_{n}^{d}$ be any truncatable stacked polytope and choose a facet $F$. As $P_{n}^{d}$ is simplicial there are precisely $d$ cutting hyperplanes $H_{i}, 1 \leq i \leq d$, that intersect this facet $F$. Place the new vertex $v$ beyond $F^{\prime}=F \cap D_{1}\left(P_{n}^{d}\right)$ and such that it is on the same side of $H_{i}$ as $D_{1}\left(P_{n}^{d}\right)$ for all $1 \leq i \leq d$. That is, choose $v$ beyond $F^{\prime}$ and beneath all other facets of $D_{1}\left(P_{n}^{d}\right)$. When we form the convex hull of $P_{n}^{d}$ and $v$, the $d$ new edges adjacent to $v$ will intersect all $d$ hyperplanes $H_{i}$. We can choose the hyperplane through these $d$ points as the cutting hyperplane for $v$. This gives a realization of $D_{1}\left(P_{n+1}^{d}\right)$.

In the construction for Theorem 3.5, we can choose the vertices of $P_{0}^{d}$ to be rational and take cutting hyperplanes with rational normal vector. Further, we can in all steps choose the vertices we add to have rational coordinates. Thus the intersection points of the cutting hyperplanes with the edges will also be rational and we obtain the following corollary.
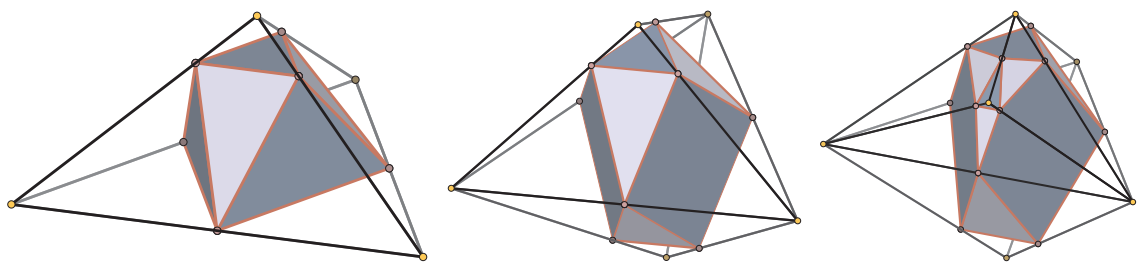

Fig. 3. Truncations of $P_{0}^{3}, P_{1}^{3}$ and $P_{2}^{3}$. 
Corollary 3.6. There are infinitely many combinatorially distinct rational 2-simplicial 2-simple 4-polytopes $D_{1}\left(P_{n}^{4}\right), n \geq 0$, with the essential components $\left(f_{0}, f_{1}, f_{2}, f_{3} ; f_{03}\right)$ of the flag vectors given by

$$
f\left(D_{1}\left(P_{n}^{4}\right)\right)=(10+4 n, 30+18 n, 30+18 n, 10+4 n ; 50+26 n) .
$$

According to Eppstein et al. [6, Proposition 8], the simplicial 4-polytope $P_{n}^{4}$ has an edge-tangent realization if and only if $n \leq 1$; this demonstrates that our vertex cutting approach is indeed much more flexible than the approach via edge-tangent realizations in [6]. The examples that we reproduce here are the hypersimplex for $n=0$, and the glued hypersimplex of Braden [3], which we get as $D_{1}\left(P_{1}^{4}\right)$.

A similar infinite sequence of rational 2-simple, 2-simplicial 4-polytopes may be obtained from a stack of $n \geq 1$ cross-polytopes. Using appropriate coordinates we obtain a realization that is rational and has the symmetries of a regular 3 -simplex. Thus we obtain a simplicial polytope $C_{n}^{4}$, with flag vector $f\left(C_{n}^{4}\right)=(4+4 n, 6+18 n, 4+$ $28 n, 2+14 n ; 8+56 n)$. Using the symmetry of the polytope and induction on $n$, it is easy to verify that $C_{n}^{4}$ in a suitable realization is indeed truncatable.

Corollary 3.7. There is an infinite sequence of rational 2-simplicial 2-simple 4-polytopes $D_{1}\left(C_{n}^{4}\right), n \geq 1$, with flag vectors

$$
f\left(D_{1}\left(C_{n}^{4}\right)\right)=(6+18 n, 12+84 n, 12+84 n, 6+18 n ; 24+120 n) .
$$

We leave the formal proof (and the explicit construction) to the reader, and instead present graphics for the construction of $D_{1}\left(C_{2}^{3}\right)$ (see Fig. 4).

\subsection{Realizations via Hyperbolic Geometry}

Now we extend the $E$-construction of [6] to higher-dimensional polytopes. In dimension $d=4$ and for $t=1$ our construction will coincide with this $E$-construction. The main goal of this section is to prove the following result.
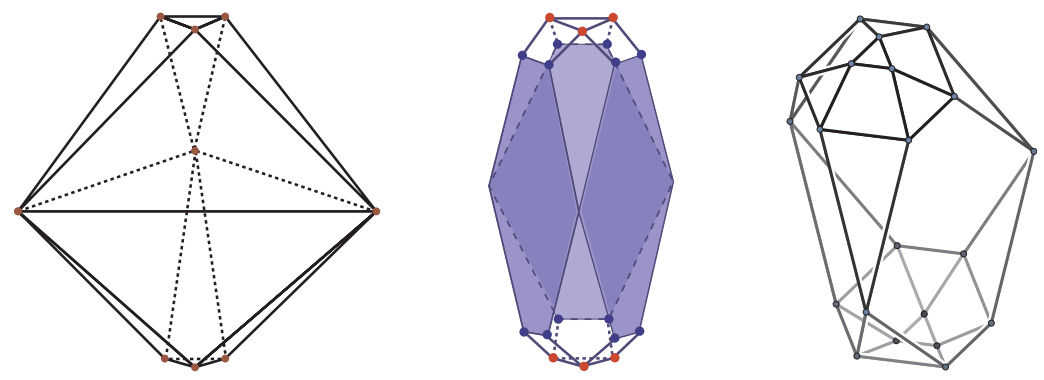

Fig. 4. Construction of $D_{1}\left(C_{2}^{3}\right)$ : the left figure shows $C_{2}^{3}$; the center figure is obtained when three vertices have been truncated; the right figure displays $D_{1}\left(C_{2}^{3}\right)$. 
Theorem 3.8. For every $d \geq 3$ there are infinitely many combinatorially distinct $(d-2)$-simplicial 2-simple d-polytopes.

The construction will roughly be as follows. First we prove that for any $d$-polytope that has its $t$-faces $(0 \leq t \leq d-1)$ tangent to the unit sphere $\mathbb{S}^{d-1}$ we can obtain a geometric realization of $E_{t}(P)$ by taking the convex hull of the vertices of $P$ and its dual. This is interesting mainly for the case $t=d-3$ and simplicial $d$-polytopes, when Corollary 2.3 tells us that $E_{d-3}(P)$ will be $(d-2)$-simplicial and 2-simple. In a next step we prove that there are in fact infinitely many combinatorially different simplicial $d$-polytopes in any dimension $d \geq 4$ that have their $(d-3)$-faces tangent to $\mathbb{S}^{d-1}$.

The theorem is trivial in dimension $d=3$, as there are infinitely many simple 3polytopes. All but the last step of our construction will also work in dimension 3, so from now on we focus on dimensions $d \geq 4$, but all illustrations refer to dimension 3 .

Theorem 3.9. Let $P$ be a d-polytope and let $1 \leq t \leq d-2$. If $P$ has its $t$-faces tangent to the unit sphere $\mathbb{S}^{d-1}$ and $P^{*}$ is the polar of $P$, then $Q:=\operatorname{conv}\left(P \cup P^{*}\right)$ is a polytopal realization of $E_{t}(P)$.

Proof. If a $t$-face $F$ of $P$ is tangent to $\mathbb{S}^{d-1}$ in a point $x \in F$, then all vertices of $F$ lie in the tangent space $T_{x} \mathbb{S}^{d-1}$ of $\mathbb{S}^{d-1}$. By the definition of the polar polytope $P^{*}$ of $P$, the face $F^{\circ}$ of $P^{*}$ dual to $F$ is also contained in $T_{x} \mathbb{S}^{d-1}$ and is orthogonal to $F$ in $T_{x} \mathbb{S}^{d-1}$. Thus the convex hull $B(F)$ of $F$ and $F^{\circ}$ has dimension $d-1$ and is tangent to $\mathbb{S}^{d-1}$ in $x$ (see Fig. 5). From this we see that $Q=\operatorname{conv}\left(P \cup P^{*}\right)$ has the orthogonal sums $B(F):=\operatorname{conv}\left(F \cup F^{\circ}\right)$ as facets.

There is a facet $B(F)$ of $Q$ corresponding to each $t$-face $F$ of $P$. The vertices of $B(F)$ are given on the one hand by the vertices of $F$, and on the other hand by the vertices of $F^{\circ}$, which are dual to the facets of $P$ that contain $F$.

Finally, one has to check that all facets of $Q$ are sums of the type $B(F)$. For this we show that all facets sharing a ridge with a facet $B(F)$ for a $t$-face $F$ of $P$ are of the type $B\left(F^{\prime}\right)$ for some $t$-face $F^{\prime}$ of $P$. The facets of $B(F)$ are the convex hulls of a facet of $F$ and a facet of $F^{\circ}$, that is, of a $(t-1)$-face $R_{F} \subset F$ of $P$ and of a $(d-t-2)$-face of $P^{*}$

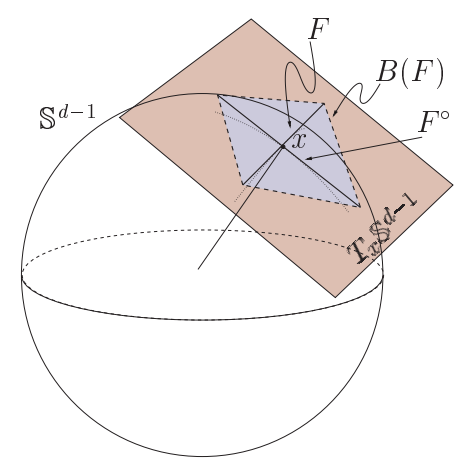

Fig. 5. A $t$-face and its polar. 
that is dual to a $(t+1)$-face $R^{F} \supset F$ of $P$. As the face lattice of $P$ is Eulerian, there is precisely one other $t$-face $F^{\prime}$ of $P$ in the interval $\left[R_{F}, R^{F}\right]$ of length 2. $B\left(F^{\prime}\right)$ also has $\operatorname{conv}\left(R_{F} \cup R^{F}\right)$ as a ridge, by construction.

Thus $Q$ has a facet $B(F)$ for every $t$-face $F$ of $P$ (and no other facets) and it has the same vertex-facet incidences as $E_{t}(P)$.

In view of Theorem 3.9, to prove Theorem 3.8 we need to construct infinitely many simplicial polytopes whose $(d-3)$-faces are tangent to the unit sphere $\mathbb{S}^{d-1}$. This is an extension of arguments that were given in [6], so we only sketch the proof and point out the differences.

We view the interior $\mathbb{D}^{d}$ of the unit sphere $\mathbb{S}^{d-1}$ as the Klein model of hyperbolic space $\mathbb{H}^{d}$, and the unit sphere $\mathbb{S}^{d-1}$ itself as the boundary at infinity $H_{\infty}^{d}$. (See, e.g. [11, p. 75] or [14].) The advantage of this model of hyperbolic space in our situation is that hyperbolic hyperplanes are the intersections of the ball with Euclidean affine planes.

Now, for simplicity, we define a $T$-polytope to be a simplicial $d$-polytope that has its $(d-3)$-faces tangent to the unit sphere $\mathbb{S}^{d-1}$. The facets and ridges of a $T$-polytope $P$ properly intersect $\mathbb{H}^{d}$, the $(d-3)$-faces are tangent to $\mathbb{S}^{d-1} \widehat{=} \mathbb{H}_{\infty}^{d}$ and all lowerdimensional faces lie outside the sphere. Thus the intersection $P^{\text {hyp }}:=\mathbb{H}^{d} \cap P$ is a convex unbounded hyperbolic polyhedron in $\mathbb{H}^{d}$.

We repeat here a caveat from [6]: A hyperideal hyperbolic object—even a convex polytope - can be positioned in such a way that it is unbounded as a Euclidean object (see p. 508 of [15]). However, we have the following lemma which can be generalized to our situation (see also [16]):

Lemma 3.10 [6, Lemma 6]. For any edge-tangent convex polytope $Q$ in $\mathbb{R}^{d}$ whose points of tangency do not lie in a hyperplane there is a hyperbolic isometry $h$ whose extension to $\mathbb{R}^{d}$ maps $Q$ into a bounded position.

We want to compute the dihedral angle between any two adjacent facets of a $T$ polytope $P$. As ridges and facets do at least partially lie inside $\mathbb{H}^{d}$, this angle is well defined as a hyperbolic dihedral angle of the hyperbolic polyhedron $P^{\text {hyp }}$ in $\mathbb{H}^{d}$ and is strictly between 0 and $\pi$.

The regular simplex and the regular cross polytope can be scaled so that their $(d-3)$ faces are tangent to the unit sphere. Let $\Delta_{d}^{\text {hyp }}$, resp. $C_{d}^{\text {hyp }}$, denote such realizations.

Lemma 3.11. The dihedral angle between any two adjacent facets of $\Delta_{d}^{\mathrm{hyp}}$, resp. $C_{d}^{\mathrm{hyp}}$, is $\pi / 2$, resp. $\pi / 3$.

Proof. This is straightforward from the arguments given in [6] when you keep in mind that their edges are in fact (codimension 3)-faces for the purpose of our proof.

Proof of Theorem 3.8. We glue together certain $T$-polytopes along facets to get new $T$-polytopes. For this we have to position the $T$-polytopes in such a way that the two 


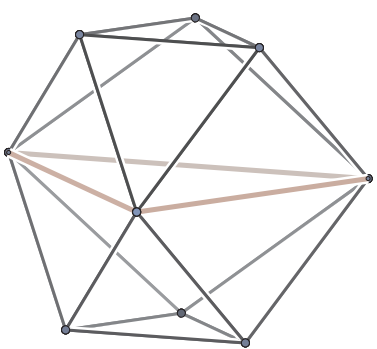

(a)

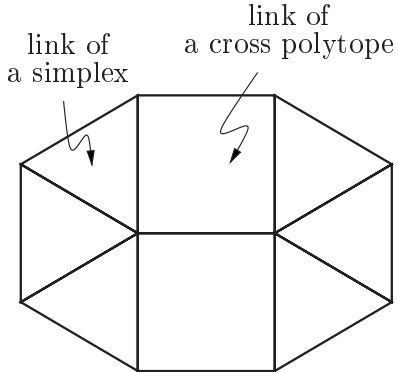

(b)

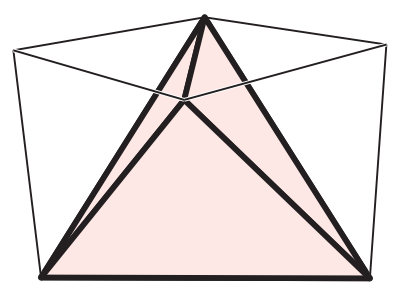

(c)

Fig. 6. The gluing construction. (a) Two glued cross polytopes, with bipyramid facets. (b) The link, with added simplices. (c) Three hyperideal tetrahedra have a bipyramidal facet (the top facet).

facets in question match, they both remain $T$-polytopes, and such that the dihedral angle between any two facets that become adjacent after the gluing is less than $\pi$ (that is, the resulting compound should be convex).

The isometry group of $\mathbb{H}^{d}$ is transitive and thus we can always map a facet of a regular simplicial $T$-polytope onto any facet of any other regular simplicial $T$-polytope. Thus gluing two such polytopes is always possible (when interpreted as hyperbolic objects).

Now we glue together two cross polytopes along a facet $F$. As the dihedral angle of two adjacent facets of a cross polytope is $\pi / 2$, the resulting polytope will have facets that are bipyramids (compare the shaded ridges in the stack of two glued cross polytopes drawn in Fig. 6(a)), but we can repair this by gluing three simplices along any of these ridges in a way illustrated in Fig. 6(b). See Fig. 6(c) for an example of three hyperideal simplices and the bipyramidal facet which we use for the gluing. The dihedral angle between two simplices is $2 \pi / 3$, and between a simplex and a cross polytope facet it is $5 \pi / 6$. Two such triples of glued-in simplices do not share a ridge if we are in dimension $d \geq 4$.

Iterating this construction by gluing $n$ cross polytopes "end-to-end" and gluing triples of simplices to all ridges having straight adjacent facets yields simplicial $T$-polytopes $Q_{n}^{d}$ with $f$-vectors

$$
\begin{aligned}
& f_{j}\left(Q_{n}^{d}\right) \\
& \quad= \begin{cases}2^{d} n-2(n-1)+d(3 d-5)(n-1) & \text { for } j=d-1, \\
2^{d-1} d n-d(n-1)+\frac{1}{2} d^{2}(3 d-5)(n-1) & \text { for } j=d-2, \\
2^{j+1}\left(\begin{array}{c}
d \\
j+1
\end{array}\right) n-\left(\begin{array}{c}
d \\
j+1
\end{array}\right)(n-1)+d\left\{3\left(\begin{array}{c}
d \\
j
\end{array}\right)-\left(\begin{array}{c}
d-1 \\
j
\end{array}\right)\right\}(n-1) & \text { for } 0 \leq j \leq d-2,\end{cases}
\end{aligned}
$$


for $n \geq 1$. From these we then derive ( $d-2)$-simplicial 2-simple $d$-polytopes $E_{d-3}\left(Q_{n}^{d}\right)$ whose $f$-vectors are given by (1) as (recall $d \geq 4$ )

$$
\begin{aligned}
f_{k}\left(E_{d-3}\left(Q_{n}^{d}\right)\right) & \begin{cases}f_{d-3}\left(Q_{n}^{d}\right) & \text { for } k=d-1, \\
\left(\begin{array}{c}
d-1 \\
2
\end{array}\right) f_{d-2}\left(Q_{n}^{d}\right) & \text { for } k=d-2, \\
\left(\begin{array}{c}
d-1 \\
3
\end{array}\right) f_{d-2}\left(Q_{n}^{d}\right)+\left(\begin{array}{c}
d \\
3
\end{array}\right) f_{d-1}\left(Q_{n}^{d}\right) & \text { for } k=d-3, \\
\left(\begin{array}{c}
d-1 \\
d-k
\end{array}\right) f_{d-2}\left(Q_{n}^{d}\right)+\left(\begin{array}{c}
d \\
d-k
\end{array}\right) f_{d-1}\left(Q_{n}^{d}\right)+f_{k}\left(Q_{n}^{d}\right) & \text { for } 1 \leq k \leq d-4, \\
f_{d-1}\left(Q_{n}^{d}\right)+f_{0}\left(Q_{n}^{d}\right) & \text { for } k=0 .\end{cases}
\end{aligned}
$$

For $d=4$ this formula indeed specializes to the $f$-vectors $(54 n-30,252 n-156,252 n-$ $156,54 n-30)$ of the four-dimensional examples that were already constructed and computed in [6].

The polytopes $E_{d-3}\left(Q_{n}^{d}\right)$ constructed in this proof have nonrational coordinates, and there seems to be no easy way to remedy this.

\subsection{Previously Known Examples}

Practically all examples of $(d-2)$-simplicial 2-simple $d$-polytopes $(d \geq 4)$ that appear in the literature may be seen as special instances of the $E_{t}$-construction for spheres as presented above, and realized by one of the two constructions of this section. In particular, the Eppstein-Kuperberg-Ziegler [6] examples arise in the special case where $P$ is a simplicial 4-polytope with an edge-tangent realization $(t=1)$, or equivalently a simple 4-polytope with a ridge-tangent realization $(t=2)$. Most of the other, earlier examples specialize even further to the case of regular polytopes $P$, where the $t$-face tangency conditions can simply be enforced by scaling — but would typically yield irrational coordinatizations, with no apparent degrees of freedom.

Until very recently, only finitely many $(d-2)$-simplicial 2-simple $d$-polytopes were known for every fixed $d \geq 4$. For $d=4$, in addition to the simplex $\Delta_{4}$ this includes a few "well-known" examples:

- Schläfli's self-dual 24-cell, with the flag vector $(24,96,96,24 ; 144)$, arises as $E_{2}(4$-cube $)=E_{1}$ (4-cross polytope). In particular, a 24-cell arose as $D_{1}\left(C_{1}^{4}\right)$ in Corollary 3.7.

- The so-called hypersimplex is a 4-polytope with a vertex-transitive automorphism group, five octahedra and five tetrahedra as facets, and flag vector $(10,30,30,10$; 50). It arises most easily as $D_{1}\left(\Delta_{4}\right)=D_{1}\left(P_{0}^{4}\right)$; see Corollary 3.6. Its dual (with an automorphism group that is transitive on the ten bipyramid facets) is thus given by $E_{1}\left(\Delta_{4}\right)$.

A much less obvious example is the glued hypersimplex of Braden [3], which arises as $D_{1}\left(P_{1}^{4}\right)$, by Corollary 3.6. 
There were claims by Perles and Shephard (see pp. 82 and 170 of [9]) for infinite families of 2-simplicial 2-simple 4-polytopes that turned out to be premature.

3.3.1. Grünbaum's Examples. The 2-simplicial $(d-2)$-simple $d$-polytopes $(d \geq 4)$ of Grünbaum [9, pp. 65 and 66] are:

$$
\begin{aligned}
K_{k}^{d} & :=\left\{x \in \mathbb{R}^{d+1}: 0 \leq x_{i} \leq 1, \sum_{i=0}^{d} x_{i}=k\right\} \quad \text { for } 1 \leq k \leq d \quad \text { (hypersimplices), } \\
M^{d} & :=\left\{x \in \mathbb{R}^{d}:\left|x_{i}\right| \leq 1, \sum_{i=1}^{d}\left|x_{i}\right| \leq d-2\right\}
\end{aligned}
$$

Since $K_{k}^{d} \cong K_{d+1-k}^{d}$, there are only $\lfloor d / 2\rfloor$ combinatorial types of hypersimplices $K_{k}^{d}$ for each dimension $d \geq 4$. Grünbaum also gives the following example of a $(d-3)$ simplicial 3-simple $d$-polytope, $d \geq 4$ :

$$
N^{d}:=\left\{x \in \mathbb{R}^{d}: \sum_{i=1}^{d} \varepsilon_{i} x_{i} \leq d-2, \varepsilon_{i}= \pm 1, \#\left\{\varepsilon_{i}=1\right\} \text { odd }\right\} \quad \text { (half-cubes). }
$$

$\Delta_{d}$ clearly has a geometric realization in which all $t$-faces are tangent to the unit sphere for any $0 \leq t \leq d-1$. Also $N_{d}$ in the given realization has its $(d-2)$-faces tangent to a sphere. The above theorem shows that we can apply the $E_{t}$-construction and an easy check proves the following combinatorial equivalences:

$$
\begin{aligned}
& \left(K_{k}^{d}\right)^{*} \cong E_{k-1}\left(\Delta_{d}\right), \quad \text { that is, } \quad K_{k}^{d} \cong D_{k-1}\left(\Delta_{d}\right) \quad \text { for } 1 \leq k \leq d, \quad \text { and } \\
& \left(M^{d}\right)^{*} \cong E_{d-2}\left(N^{d}\right), \quad \text { that is, } \quad M^{d} \cong D_{d-2}\left(N^{d}\right) \cong D_{1}\left(N^{d}\right)^{*},
\end{aligned}
$$

with the obvious generalization $D_{k}$ of vertex truncation that preserves only one (relative interior) point from each $k$-face. The polytope $N^{d}$ itself cannot be a result of an $E_{t^{-}}$construction, as for $d \geq 6$ the polytope $N^{d}$ and its dual are at least 3-simple.

3.3.2. The Gosset-Elte Polytopes and Wythoff's Construction. Recently, Peter McMullen [9] noted that the Gosset-Elte polytopes described in the classic book of Coxeter [4] are $(r+2)$-simplicial and $(s+t-1)$-simple. The Gosset-Elte polytopes $r_{s t}$ for $r, s, t \geq 0$ arise from the Wythoff construction in the following way: Consider the group of reflections corresponding to the diagram in Fig. 7, where we have $r$ nodes on the right end, $s$ nodes on the left end and $t$ nodes on the lower end. The group of reflections is finite if and only if $1 /(r+1)+1 /(s+1)+1 /(t+1)>1$. This leaves us with only three infinite series for $r, s$ and $t$ and a finite number of other choices.

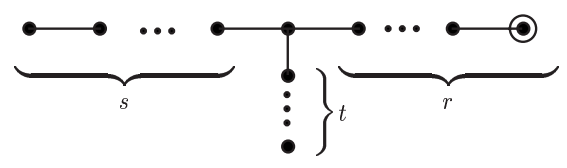

Fig. 7. The Gosset-Elte polytopes. 
Table 1. Examples of $E_{t}$-polytopes that were known before.

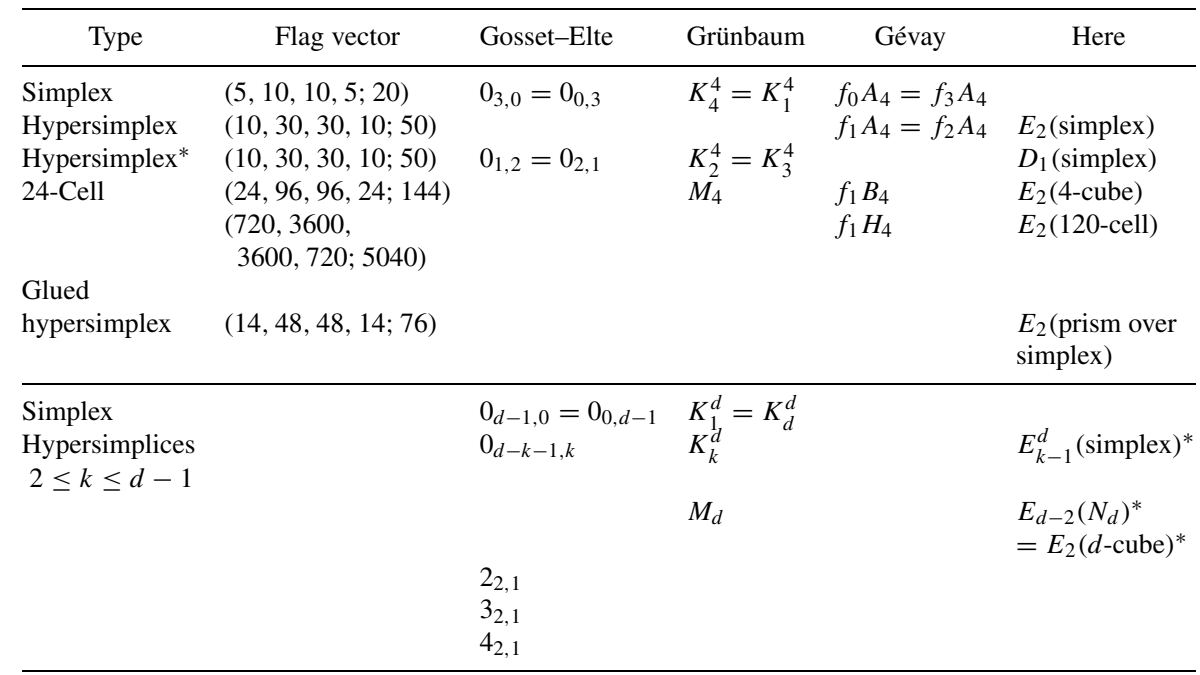

The three infinite series are $0_{d-k, k-1}$ for $1 \leq k \leq d, 1_{d-3,1}$ and $(d-3)_{1,1}$. The first two coincide with Grünbaum's examples $K_{k}^{d}$ and $N^{d}$, while the last one gives the $d$-dimensional cross polytope. Among the remaining finite number of choices for $r, s$ and $t$, only $2_{2,1}, 3_{2,1}$ and $4_{2,1}$ are $(d-2)$-simplicial and 2-simple. Out of these, only $0_{d-k-1, k}$ for $2 \leq k \leq d-1$ is contained in our construction, as the $1_{d-3,1}$ are 3 -simple and all others have simplicial facets, which is not possible for a polytope resulting from the $E_{t}$-construction for $0<t<d-1$.

3.3.3. Gévay's Polytopes. Gévay constructs a number of interesting uniform polytopes as "Kepler hypersolids" that may be obtained as follows: take $P$ to be a regular polytope, scale it such that its $t$-faces are tangent to the unit sphere, and then let $f_{t}(P)$ be the convex hull of $P$ and its dual. The polytopes thus obtained are dual to the corresponding Wythoff/Gosset-Elte polytopes [7, p. 128].

As a specific instance, Gévay [7], [8] describes the "dipyramidal 120-cell" $f_{1} H_{4}$, which in [6] appears as $E$ (120-cell).

3.3.4. Overview. All examples except for those from [6] are collected in Table 1. The first part of the table lists four-dimensional examples, and the second part lists examples in higher dimensions. In dimension $d=3$ we have infinitely many 2-simple (i.e. simple) polytopes. The flag vector of a general 4-polytope is denoted as $\left(f_{0}, f_{1}, f_{2}, f_{3} ; f_{03}\right)$. Any 2-simplicial 2-simple 4-polytope has a flag vector of the special form $\left(f_{0}, f_{1}, f_{1}, f_{0} ; f_{1}+2 f_{0}\right)$.

\section{Some Corollaries}

While 2-simple 2-simplicial ("2s2s" for the purpose of this section) 4-polytopes seemed hard to construct until very recently (with only finitely many examples known), we 
have now achieved quite some flexibility. In particular, the vertex truncation method of Section 3.1 makes it easy to construct examples, say with specific conditions on the resulting flag vectors. To demonstrate this, we here derive a sequence of corollaries.

The $f$-vector of a $2 \mathrm{~s} 2 \mathrm{~s} 4$-polytope is necessarily symmetric $\left(f_{0}=f_{3}\right.$ and $\left.f_{1}=f_{2}\right)$, and it also determines the flag vector, with $f_{03}=f_{1}+2 f_{2}$. Thus the $f$-vectors of $2 \mathrm{~s} 2 \mathrm{~s}$ 4-polytopes have at most two independent parameters. Our first corollary shows that we do indeed need two parameters.

Corollary 4.1. There are $2 s 2 s$ 4-polytopes that have the same numbers of vertices (and facets), but that have different numbers of edges (and ridges).

Proof. The four-dimensional regular cross polytope $C^{*}$ is clearly truncatable. Furthermore, we can stack 4-simplices onto nine out of its sixteen facets, so that the resulting simplicial 4-polytope with $f$-vector $(17,60,86,43)$ is truncatable. Thus we obtain a 2s2s 4-polytope $D_{1}\left(C^{*}\right)$ with $f$-vector $(60,258,258,60)$.

On the other hand, vertex truncation of a stack of three cross polytopes as in Corollary 3.7 yields $D_{1}\left(C_{3}^{4}\right)$ with $f$-vector $(60,264,264,60)$.

Corollary 4.2. The number of combinatorially distinct $2 s 2 s$ 4-polytopes with the $f$ vector $f\left(D_{1}\left(P_{n}^{4}\right)\right)=(10+4 n, 30+18 n, 30+18 n, 10+4 n ; 50+26 n)$ grows exponentially with $n$.

Proof. There are exponentially many stacked 4-polytopes with $n+5$ vertices. This already follows from the fact that there are exponentially many (unlabelled) trees of maximal degree 5 on $n+1$ vertices.

Thus we need to see that the combinatorial type of any stacked 4-polytope $P_{n}^{4}$ can be reconstructed from its vertex truncation $D_{1}\left(P_{n}^{4}\right)$. (See Corollary 3.6.) The facets of $D_{1}\left(P_{n}^{4}\right)$ are on the one hand truncated simplices $F^{\prime}$, which are octahedra, and on the other hand the vertex figures $F_{v}$ of $P_{n}^{4}$, which are stacked. Furthermore, two of the octahedra $F^{\prime}$ and $G^{\prime}$ are adjacent if and only if the corresponding facets $F$ and $G$ of $P_{n}^{4}$ are adjacent. Thus we get from $D_{1}\left(P_{n}^{4}\right)$ the dual graph of $P_{n}^{4}$, which determines the combinatorial type of $P_{n}^{4}$ by the reconstruction theorem of Blind and Mani [22, Section 3.4].

One can tell from the flag vector whether a polytope is 2-simplicial, since this amounts to the condition $f_{02}=3 f_{2}$, and similarly for 2-simplicity. Our next corollary shows that there is no similar criterion to derive this information from the $f$-vector.

Corollary 4.3. $\quad A 2 s 2 s$ and a not-2s $2 s$ 4-polytope can have the same $f$-vector.

Proof. Using a hyperbolic gluing construction for a stack of $n$ 600-cells, Eppstein et al. [6, Section 3.3] produced simplicial edge-tangent 4-polytopes $Q_{n}$ with $f$-vectors $(106 n+14,666 n+54,666 n+54,106 n+14)$ and thus 2s2s 4-polytopes $E_{1}\left(Q_{n}\right)$ with $f$ vector $(54+666 n, 240+3360 n, 240+3360 n, 54+666 n)$. We take this for $n=13$, that is, we get $f\left(E_{1}\left(Q_{13}\right)\right)=(8712,43920,43920,8712)$ for a polytope that has lots of facets that are bipyramids over pentagons, and lots of "regular" vertices that are contained 
in exactly 12 such bipyramids, with a dodecahedral vertex figure. Now truncating 80 such "regular vertices" and stacking pyramids over the resulting dodecahedral facets as well as onto 80 other bipyramidal facets that are not involved in this results in a not-2s2s 4-polytope $E_{1}\left(Q_{13}\right)^{\prime}$ with $f\left(E_{1}\left(Q_{13}\right)^{\prime}\right)=(10392,48280,48480,10392)$.

The 2s2s 4-polytope $D_{1}\left(C_{577}^{4}\right)$ of Corollary 3.7 has exactly the same $f$-vector.

In the investigations of Eppstein et al. [6], 2s2s 4-polytopes were of interest as they provided examples of 4-polytopes for which the "fatness" parameter $\Phi(P):=$ $\left(f_{1}+f_{2}\right) /\left(f_{0}+f_{3}\right)$ is particularly large. We now produce two 4-polytopes on the same number of vertices, one of them not-2s $2 \mathrm{~s}$, where the $2 \mathrm{~s} 2 \mathrm{~s}$ example is less fat.

Corollary 4.4. There is a $2 s 2 s$ 4-polytope with the same number of vertices and facets as a 4-polytope that is not $2 s 2 s$ but has fewer edges and ridges.

Proof. The "dipyramidal 720-cell" $E:=E_{2}(120$-cell $)=D_{1}(600 \text {-cell })^{*}$ as discussed above has $f$-vector $(720,3600,3600,720)$. Now we perform some operations that destroy 2-simplicity and 2-simpliciality: we truncate two vertices with a dodecahedral vertex figure, and stack pyramids on the resulting dodecahedral facets, and we also stack pyramids onto two bipyramidal facets. Thus we get a new polytope $E^{\prime}$ with $f$-vector $(762,3714,3714,762)$.

On the other hand, vertex truncation applied to a stack of 42 cross polytopes yields the 2s2s 4-polytope $D_{1}\left(C_{42}^{4}\right)$ with $f$-vector $(762,3540,3540,762)$.

\section{Open Problems}

1. Prove for some polytope $P$ and some $t$ that $E_{t}(P)$ does not have a polytopal realization.

2. Give an example of some $E_{t}(P)$ that does not have a rational realization. $E_{2}(120-$ cell) is a candidate for this.

3. Modify the $E_{t}$-construction in order to produce also $(d-3)$-simplicial 3-simple polytopes, such as the half-cubes $N^{d}$.

\section{Acknowledgement}

We thank Carsten Lange, Julian Pfeifle and Arnold Waßmer for several discussions and valuable hints.

\section{References}

1. M. M. Bayer and L. J. Billera, Generalized Dehn-Sommerville relations for polytopes, spheres and Eulerian partially ordered sets, Invent. Math., 79 (1985), 143-157.

2. A. Björner, M. Las Vergnas, B. Sturmfels, N. White, and G. M. Ziegler, Oriented Matroids, second edn., Encyclopedia of Mathematics, vol. 46, Cambridge University Press, Cambridge, 1999.

3. T. Braden, A glued hypersimplex, personal communication, 1997. 
4. H. S. M. Coxeter, Regular Polytopes, second edn., Macmillan, New York, 1963. Corrected reprint, Dover, New York, 1973.

5. H. S. M. Coxeter, Wythoff's construction for uniform polytopes, Proc. London. Math. Soc. (2), 38 (1935), 327-339; reprinted in: Twelve Geometric Essays, Southern Illinois Press, Carbondale, IL, 1968, pp. 40-53.

6. D. Eppstein, G. Kuperberg, and G. M. Ziegler, Fat 4-polytopes and fatter 3-spheres, in Discrete Geometry: In honor of W. Kuperberg's 60th birthday, A. Bezdek, ed., Pure and Applied Mathematics, vol. 253, Marcel Dekker, New York, 2003, pp. 239-265. arXiv:math.CO/0204007.

7. G. Gévay, Kepler hypersolids, in Intuitive Geometry (Szeged, 1991), Colloquia Mathematica Societati János Bolyai, vol. 63, North-Holland, Amsterdam, 1994, pp. 119-129.

8. G. Gévay, On perfect 4-polytopes. Beiträge Algebra Geom. 43 (2002), 243-259.

9. B. Grünbaum, Convex Polytopes, Graduate Texts in Mathematics, vol. 221, Springer-Verlag, New York, 2003. Second edition edited by V. Kaibel, V. Klee, and G. M. Ziegler. (Original edition: Interscience, London, 1967.)

10. J. F. P. Hudson, Piecewise Linear Topology, Benjamin, New York, 1969.

11. B. Iversen, Hyperbolic Geometry, London Mathematical Society Student Texts, vol. 25, Cambridge University Press, Cambridge, 1992.

12. G. Kalai, Polytope skeletons and paths, in Handbook of Discrete and Computational Geometry, J. E. Goodman and J. O'Rourke, eds., Chapman \& Hall/CRC Press, Boca Raton, FL, second edn., 2004, chapter 20, pp. 455-476.

13. V. Klee, A combinatorial analogue of Poincaré's duality theorem, Canad. J. Math., 16 (1964), 517-531.

14. J. G. Ratcliffe, Foundations of Hyperbolic Manifolds, Graduate Texts in Mathematics, vol. 149, SpringerVerlag, New York, 1991.

15. E. Schulte, Analogues of Steinitz's theorem about non-inscribable polytopes, in Intuitive Geometry (Amsterdam, 1985), Colloquia Matematica Societati János Bolyai, vol. 48, North-Holland, Amsterdam, 1987, pp. 503-516.

16. B. Springborn, A unique representation theorem of polyhedral types: Centering via Möbius transformations, Math Z., to appear. arXiv:math.MG/0401005.

17. R. P. Stanley, Some aspects of groups acting on finite posets, J. Combinatorial Theory Ser. A, 32 (1982), 132-161.

18. R. P. Stanley, Enumerative Combinatorics, Vol.I, Wadsworth and Brooks/Cole, Monterey, CA, 1986. Second edn., Cambridge Studies in Advanced Mathematics, vol. 49, Cambridge University Press, Cambridge, 1997.

19. R. P. Stanley, Flag $f$-vectors and the $c d$-index, Math. Z., 216 (1994), 483-499.

20. R. P. Stanley, A survey of Eulerian posets, Proc. NATO Advanced Study Institute on "Polytopes: Abstract, convex and computational" (Toronto, 1993), T. Bisztriczky, P. McMullen, and A. Weiss, eds., Kluwer, Dordrecht, 1994, pp. 301-333.

21. J. W. Walker, Canonical homeomorphisms of posets, European J. Combin., 9 (1988), 97-108.

22. G. M. Ziegler, Lectures on Polytopes, Graduate Texts in Mathematics, vol. 152, Springer-Verlag, New York, 1995; revised edn. 1998.

23. G. M. Ziegler, Face numbers of 4-polytopes and 3-spheres, Proceedings of the International Congress of Mathematicians (ICM 2002, Beijing), vol. III, L. Tatsien, ed., Higher Education Press, Beijing, 2002, pp. 625-634. arXiv:math.MG/0208073.

Received May 7, 2003, and in revised form March 17, 2004. Online publication August 23, 2004. 